\title{
Two cases of atypical membranoproliferative glomerulonephritis showing opposite clinical course
}

\author{
Yuya Hashimura $\cdot$ Hiroshi Kaito $\cdot$ Kandai Nozu \\ Yuko Shima $\cdot$ Koichi Nakanishi $\cdot$ Kazumoto Iijima • \\ Norishige Yoshikawa
}

Received: 2 September 2011/Accepted: 20 February 2012/Published online: 10 April 2012

(C) Japanese Society of Nephrology 2012

\begin{abstract}
Atypical membranoproliferative glomerulonephritis (MPGN) is considered to progress to typical MPGN, and it is believed that it can be treated with corticosteroids. However, consensus that atypical MPGN is a continuum of morphologic manifestations of typical MPGN cannot be reached. Herein, we report two cases of atypical MPGN with opposite clinical course. Case 1 was a 4-year-old boy with macrohematuria and proteinuria with no prodromal symptoms. His serum C3 level had abruptly dropped, and renal biopsy confirmed a diagnosis of atypical MPGN. After performing kidney biopsy, his urinary abnormality improved and his C3 level had normalized 1 year after onset without medication. At the most recent follow-up, neither proteinuria nor hematuria was detected. Case 2 was a 7-year-old girl with microhematuria and proteinuria at her school urinary screening. Her first biopsy finding was similar to dense deposit disease, and the second biopsy showed atypical MPGN. Oral corticosteroids were started from this point, but heavy proteinuria and hypocomplementemia could not be improved sufficiently. We immediately performed third kidney biopsy and diagnosed typical MPGN. These findings suggest that the indication of therapy for atypical MPGN should be re-examined. Aggressive therapy such as steroid administration is not necessarily essential and effective for therapeutic intervention of all atypical MPGN. Moreover, atypical MPGN
\end{abstract}

Y. Hashimura · H. Kaito $(\bowtie) \cdot$ K. Nozu · K. Iijima

Department of Pediatrics, Kobe University Graduate

School of Medicine, 7-5-1 Kusunoki-cho, Chuo,

Kobe, Hyogo 6500017, Japan

e-mail: hkaitoh@med.kobe-u.ac.jp

Y. Shima $\cdot$ K. Nakanishi $\cdot$ N. Yoshikawa

Department of Pediatrics, Wakayama Medical University,

Wakayama, Japan may involve different etiologic and pathogenetic factors, rather than a continuum of morphologic manifestations of MPGN.

Keywords Angiotensin converting enzyme inhibitor . Corticosteroid therapy - Atypical membranoproliferative glomerulonephritis

\section{Introduction}

Atypical membranoproliferative glomerulonephritis (MPGN), which includes the concept of focal MPGN, is morphologically characterized by few or no glomeruli with segmental proliferative MPGN lesions and residual glomeruli presenting only mild to moderate cellular proliferation. A double-contoured appearance is not necessarily present in all cases, and some cases have the histological features of diffuse mesangial proliferative glomerulonephritis with positive findings of third component of complement (C3) fluorescent immunostaining [1]. Some previous reports demonstrated that atypical MPGN may progress to typical MPGN, which is why it is believed to be treatable with corticosteroids. However, consensus cannot be reached that atypical MPGN is an early type of typical MPGN and that patients with atypical MPGN are candidates for administration of corticosteroids. Herein, we report two children with atypical MPGN showing opposite clinical course.

\section{Case 1}

A 4-year-old boy without any past history developed macrohematuria and proteinuria with no prodromal 
symptoms. At the initial visit, his blood examination was completely normal including serum complement titer [C3: $76 \mathrm{mg} / \mathrm{dl}$ (normal 65-135 mg/dl) and C4: $28 \mathrm{mg} / \mathrm{dl}$ (normal 17-33 mg/dl)], antistreptolysin O (ASO) titer, antinuclear antibody, and hepatitis $\mathrm{B}$ and $\mathrm{C}$ virus antibody. We followed him closely without therapy. Five months after onset, his serum C3 level had abruptly dropped to $32 \mathrm{mg} / \mathrm{dl}$ and could not return toward normal. Seven months after onset, percutaneous kidney biopsy was performed with histological signs of atypical MPGN (Table 1). Light microscopy showed diffuse mesangial proliferation, but double-contoured appearance was not found (Fig. 1a, b). Immunofluorescent staining for C3 was positive only in the mesangial lesion and along the capillary walls, which showed a fringe pattern (Fig. 1c). Electron microscopy showed partial mesangial interposition and electron-dense deposits at mesangial and subendothelial lesions (Fig. 1d). At time of kidney biopsy, his proteinuria had almost disappeared. Therapeutic intervention was not provided, as his urinary abnormality was gradually improving without medication. His C3 level had normalized 1 year after onset, and at the most recent follow-up (2 years after onset), neither abnormal urinalysis nor hypocomplementemia was detected (Fig. 2). Furthermore, his renal function and blood pressure were normal.

\section{Case 2}

A 7-year-old girl visited our outpatient clinic because of microhematuria and proteinuria at her school urinary screening. She had no family history of kidney disease. At

Table 1 Light and electron microscopic findings of two patients

\begin{tabular}{|c|c|c|c|c|c|c|c|c|c|c|}
\hline \multirow[t]{3}{*}{ Patient no. } & \multirow[t]{3}{*}{ Number of biopsy } & \multicolumn{4}{|l|}{ Light microscopy } & \multicolumn{5}{|c|}{ Electron microscopy } \\
\hline & & \multirow[t]{2}{*}{ No. of glomeruli } & \multirow{2}{*}{$\begin{array}{l}\text { Mesangial } \\
\text { proliferation }\end{array}$} & \multicolumn{2}{|c|}{ Double contour } & \multicolumn{4}{|l|}{ Deposit } & \multirow[t]{2}{*}{ MI } \\
\hline & & & & Segmental & Global & Mesangial & Subendo & Subepi & Intra & \\
\hline 1 & $1 \mathrm{st}$ & 46 & Diffuse & - & - & $3+$ & $1+$ & - & - & $1+$ \\
\hline \multirow[t]{3}{*}{2} & $1 \mathrm{st}$ & 5 & Diffuse & - & - & $1+$ & - & - & $3+$ & $1+$ \\
\hline & 2 nd & 30 & Diffuse & + & - & $1+$ & $1+$ & - & - & - \\
\hline & $3 \mathrm{rd}$ & 16 & Diffuse & - & + & $1+$ & $1+$ & - & - & $1+$ \\
\hline
\end{tabular}

Subendo subendothelial, Subepi subepithelial, Intra intramembranous, MI mesangial interposition

Fig. 1 Kidney biopsy findings of case 1. a Light microscopic finding of kidney biopsy by Periodic acid-Schiff stain ( $\times 100)$. b Light microscopic finding of kidney biopsy by Periodic acid-methenaminesilver stain $(\times 400)$.

c Immunofluorescence showing deposits of $\mathrm{C} 3$ along capillary walls. d Electron microscopic finding of intramembranous dense deposits
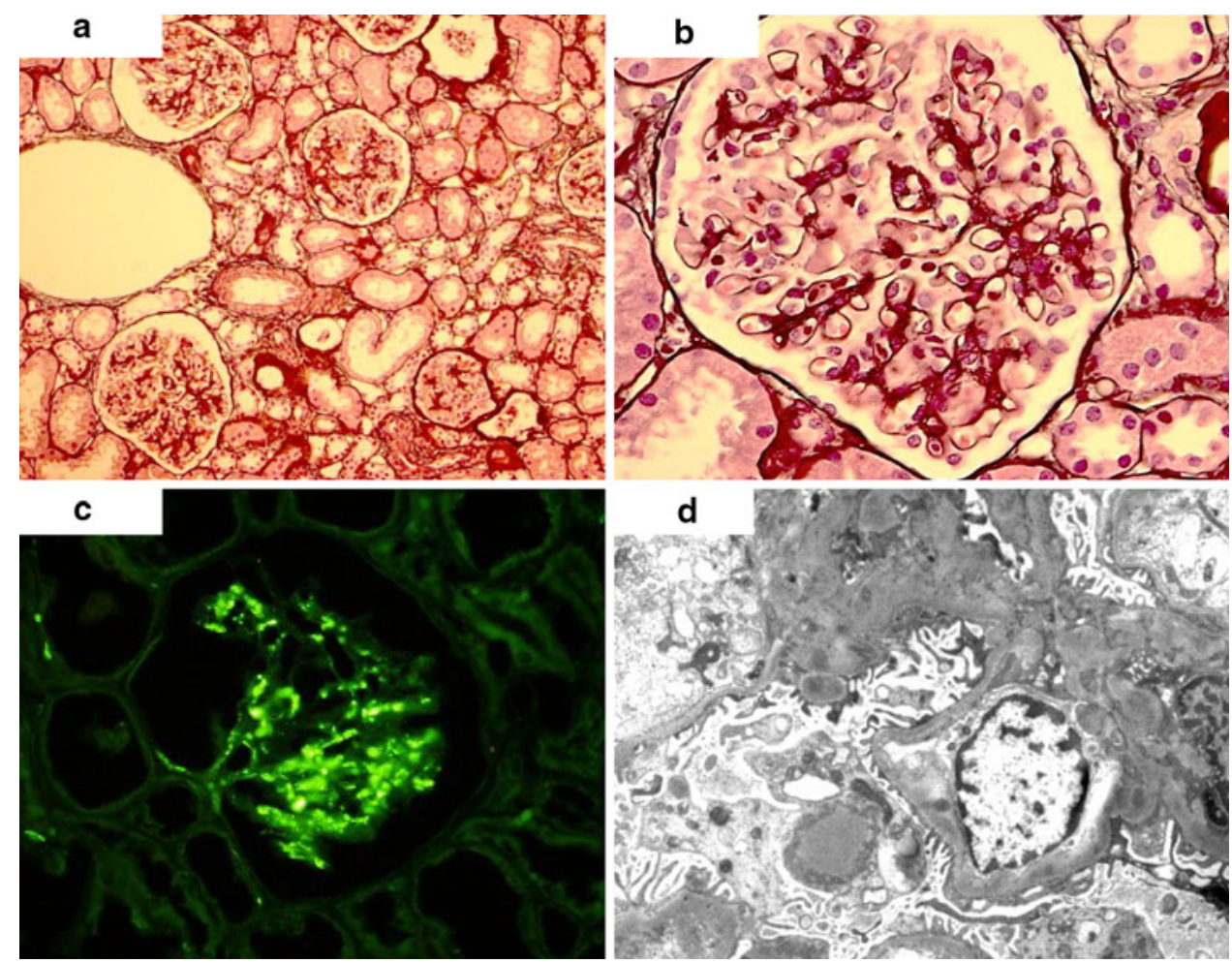
Fig. 2 Clinical course of case 1



the initial visit her urinary protein/creatinine ratio $(\mathrm{u}-\mathrm{P} / \mathrm{Cr})$ was $1.1 \mathrm{~g} / \mathrm{gCre}$. Her serum $\mathrm{C} 4$ level was decreased to $11 \mathrm{mg} / \mathrm{dl}$, with serum C3 normal. Her proteinuria persisted for more than 2 months, and we performed percutaneous kidney biopsy. Light microscopy showed diffuse mesangial proliferation (Fig. 3a). Immunofluorescent staining for C3 was positive only along the capillary walls of the glomeruli and showed a fringe pattern (Fig. 3c). Electron microscopy showed intramembranous dense deposits, which were similar to those seen in dense deposit disease (DDD), but the latter features noticeably high concentrations (Fig. 3b). We started to treat her with angiotensin-converting enzyme inhibitor (lisinopril), and her $\mathrm{u}-\mathrm{P} / \mathrm{Cr}$ was gradually decreased to $0.2 \mathrm{~g} / \mathrm{gCre}$. Thirty months after onset, her $\mathrm{u}-\mathrm{P} /$ $\mathrm{Cr}$ had increased to $2.0 \mathrm{~g} / \mathrm{gCre}$ without any blood examination abnormalities including $\mathrm{C} 3$ and $\mathrm{C} 4$ level. We immediately performed a second kidney biopsy. Light microscopy showed an increase in mesangial proliferation compared with the first biopsy. In addition, focal and segmental double-contoured appearance was found (Fig. 3d), and moreover, the intramembranous deposits seen in the first biopsy had disappeared. These findings led us to the diagnosis of atypical MPGN, and we started treatment with oral corticosteroids beginning at $80 \mathrm{mg}$ per day of prednisolone (PSL). After this therapy, her proteinuria gradually improved, and we therefore tapered and stopped PSL by 1 year after the second biopsy. Three months after the end of PSL administration, her $\mathrm{u}-\mathrm{P} / \mathrm{Cr}$ was as high as $1.7 \mathrm{~g} / \mathrm{gCre}$ and her serum C3 level was decreased to $48 \mathrm{mg} / \mathrm{dl}$. We immediately performed a third renal biopsy. Light microscopy showed severe diffuse and segmental mesangial proliferation accompanied by lobulation, capillary occlusion, and double contour of the glomerular capillary wall (Fig. 3e), which suggested typical MPGN. We initiated methylprednisolone pulse therapy three times followed by alternate-day oral PSL. After a therapeutic procedure, her serum C3 level had normalized and her proteinuria gradually disappeared. At the most recent follow-up (3.5 years after third biopsy) her blood examination was completely normal including serum complement titer and she showed neither urinary abnormalities nor renal insufficiency with administration of alternate-day oral PSL and lisinopril (Fig. 4).

\section{Discussion}

Some previous reports concluded that atypical MPGN is an early manifestation of typical MPGN [2]; these findings are based on results of some retrospective study reporting that atypical MPGN patients treated with corticosteroids had good prognosis, while those who were not treated with them progressed to typical MPGN [3]. They argue that atypical MPGN represents different points in a continuum of morphologic manifestations of MPGN. In one of our present cases, case 2, atypical MPGN progressed to typical MPGN, which partially supports these findings. The other present case, case 1, had good prognosis without medication, suggesting that the current common practice of treating all children with atypical MPGN with corticosteroids should be re-examined.

In Japan, the Ministry of Education began school urinary screening programs aimed at early detection of insidious renal diseases in 1974. Since then, some reports have demonstrated that atypical MPGN has good prognosis with oral corticosteroids. However, we previously revealed excellent prognoses for atypical MPGN patients with nonsteroidal therapy [1]. On the other hand, in case 2, atypical MPGN progressed to typical MPGN despite immediate initiation of corticosteroid therapy. The first biopsy finding of this case was similar to DDD, and a few years later it converted to typical MPGN via focal MPGN. It is of much more interest that her clinical course after diagnosis of atypical MPGN seems to be rather nonprogressive. Our case suggested that atypical MPGN may involve different etiologic and pathogenetic factors, rather than a continuum of morphologic manifestations of idiopathic MPGN. Therapeutic efficacy may depend heavily on 

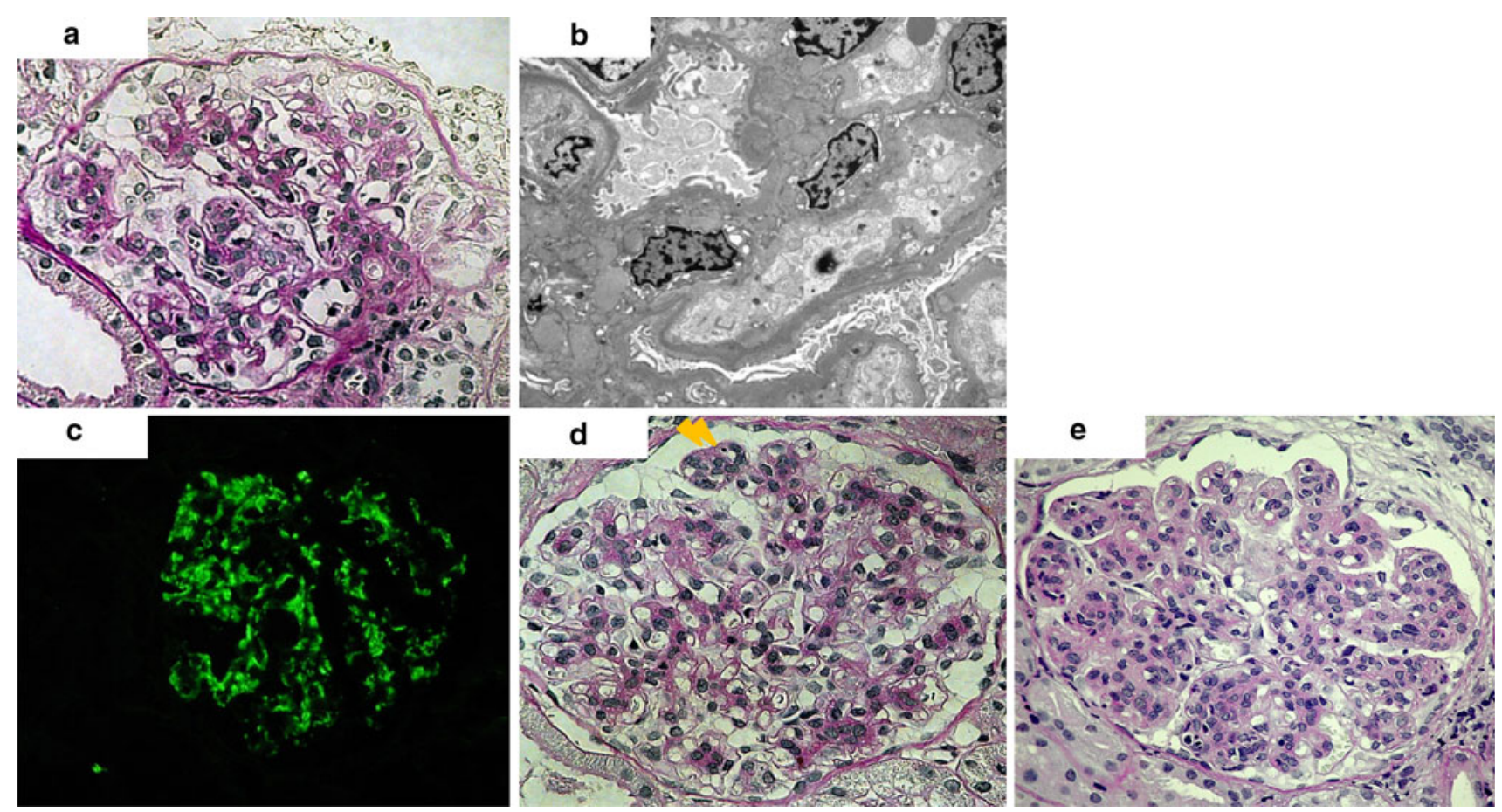

Fig. 3 Kidney biopsy findings of case 2. a Light microscopic finding of first kidney biopsy: diffuse mesangial proliferation was shown by Periodic acid-Schiff stain $(\times 400)$. b Electron microscopic finding of first kidney biopsy: intramembranous dense deposits were shown. c Immunofluorescence showing deposits of C3 along capillary walls. d Light microscopic finding of second kidney biopsy: mesangial proliferation increased and focal and segmental double-contoured appearance was found (indicated by yellow arrowhead) by Periodic acid-Schiff stain $(\times 400)$. e Light microscopic finding of third kidney biopsy: severe segmental mesangial proliferation and double contour of the glomerular capillary wall were shown by Periodic acid-Schiff stain $(\times 400)$

Fig. 4 Clinical course of case 2

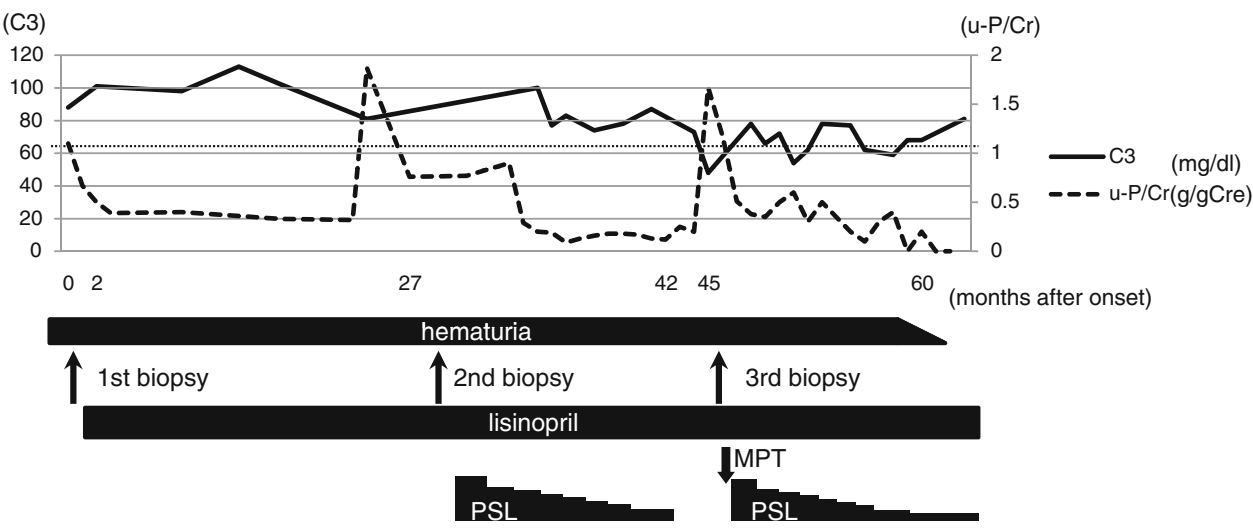

these factors. In our cases, we examined everything we could to rule out systemic and infectious disorders, but no positive results could be detected.

Little is known concerning the critical point in the progression and remission of atypical MPGN. A previous report demonstrated that some patients with low serum level of C3 for a relatively long time tend to progress to typical MPGN from atypical MPGN [4]. However, our cases indicated that histological findings in atypical MPGN cannot be correlated with the duration of low serum level of $\mathrm{C} 3$.
In conclusion, we suggest that early initiation of steroid therapy is not necessarily essential and effective for all atypical MPGN. Atypical MPGN may involve different etiologic and pathogenetic factors rather than a continuum of morphologic manifestations of MPGN, and therapeutic efficacy may depend heavily on these factors. Steroids may not be appropriate as first-line treatment of atypical MPGN, as our cases show that steroidal therapy including methylprednisolone pulse will be effective even when duration of atypical MPGN is relatively long or it progress to typical 
MPGN. Further studies are needed to confirm our arguments.

\section{References}

1. Fujita T, Nozu K, Iijima K, Kamioka I, Yoshiya K, Tanaka R, et al. Long-term follow-up of atypical membranoproliferative glomerulonephritis: are steroids indicated? Pediatr Nephrol. 2006;21:194-200.
2. Strife CF, McAdams AJ, West CD. Membranoproliferative glomerulonephritis characterized by focal, segmental proliferative lesions. Clin Nephrol. 1982;18:9-16.

3. Iitaka K, Nakamura S, Moriya S, Motoyama O, Sakai T, et al. Focal segmental membranoproliferative glomerulonephritis in children. Pediatr Nephrol. 2003;18:1000-4.

4. Hattori S, Hiramatsu M, Ushijima T, Karashima S, Furuse A, Matsuda I, et al. Clinicopathological survey of persistent hypocomplementemic glomerulonephritis in children; correlation of DPGN and MPGN type I. (in Japanese). Nippon Jinzo Gakkai Shi. 1992;34:773-81. 